\title{
Avaliação dos Sentimentos Hedônicos com a Utilização de Práticas Pedagógicas
}

\author{
Clara Berenguer Ledo \\ Universidade Salvador \\ Salvador, Bahia, Brasil \\ claraberenguerledo@gmail.com
}

\author{
Artur Henrique Kronbauer \\ Universidade do Estado da Bahia / Universidade Salvador \\ Salvador, Bahia, Brasil \\ arturhk@gmail.com
}

\begin{abstract}
RESUMO
Este artigo apresenta um estudo referente aos sentimentos hedônicos despertados nos estudantes em relação a um conjunto de metodologias ativas aplicadas nas aulas de Interação HumanoComputador. Para a coleta dos dados foi aplicada a técnica de autorrelato Affect Grid utilizada em avaliações de User eXperience (UX). O estudo foi realizado com a participação de alunos de Engenharia da Computação ao longo de três meses. Os dados capturados durante a condução do experimento permitiram identificar os sentimentos hedônicos causados aos alunos, possibilitando ao professor identificar quais metodologias ativas são mais adequadas para a geração de sentimentos positivos.
\end{abstract}

\section{PALAVRAS-CHAVE}

experiencia de usuário, metodos de UX, experiencias hedonicas, Affect Grid

\section{INTRODUÇÃO}

O termo Experiência de Usuário (User eXperience - UX) vem sendo bastante utilizado nos últimos anos por especialistas e pesquisadores das áreas de Usabilidade, Interação Homem-Computador (IHC), Ciência Cognitiva, Ergonomia, dentre outras.

A ISO 9241-210 [12] define o termo Experiência do Usuário como "as percepções e reações de uma pessoa que resultam do uso ou da utilização prevista de um produto, sistema ou serviço", influenciadas por três fatores: o usuário, o sistema e o contexto de uso. No trabalho [8], consta que a experiência do usuário engloba todas as interações e podem gerar sensações, reações e emoções positivas ou negativas. Tais sentimentos podem ocorrer antes, durante e depois da utilização de um sistema, produto ou serviço [4].

Deste modo, a importância de detectar e entender as emoções causadas pelas práticas pedagógicas utilizadas em sala de aula é significativa. Com estas informações, o docente pode adaptar as metodologias e proporcionar ao discente sentimentos positivos, potencializando o seu interesse pelos assuntos tratados em aula.

Segundo [2], as Metodologias Ativas são métodos de ensino desenvolvidos com o objetivo de centralizar a atenção no estudante durante o processo de aprendizagem. Desta forma, o discente deixa

Permission to reproduce or distribute, in whole or in part, material extracted from this work, verbatim, adapted or remixed, as well as the creation or production from the content of such work, is granted without fee for non-commercial use, provided that the original work is properly credited.

IHC 2019 - Workshop sobre Educação em IHC (WEIHC), Outubro 21-25, 2019, Vitória, Brasil. In Anais Estendidos do XVIII Simpósio Brasileiro sobre Fatores Humanos em Sistemas Computacionais. Porto Alegre: SBC

(C) 2019 by the author(s), in accordance with the terms of the Creative Commons Attribution-NonCommercial 4.0 International Public License (CC BY-NC 4.0). de ser um agente passivo que apenas escuta e torna-se um membro ativo na construção do saber. Esta mudança pode ser promovida através de estímulos provocados pelo professor para potencializar os seus pensamentos críticos e promover uma maior reflexão sobre o assunto. Desta maneira, o discente sairá da universidade preparado para as mais diversas situações presentes no mercado de trabalho.

Neste contexto, esta pesquisa tem como objetivo avaliar os sentimentos hedônicos dispertados por algumas práticas pedagógicas aplicadas na disciplina de IHC, no curso de Engenharia da Computação (EC), com a utilização da técnica Affect Grid que foi criada para avaliar a UX.

A contribuição esperada da pesquisa é apresentar uma forma de identificar os sentimentos hedônicos causados com a variação das práticas pedagógicas e permitir ao professor adequá-las para se tornarem mais atraentes aos alunos.

O restante deste artigo está estruturado da seguinte forma: na Seção 2, serão abordados conceitos de UX e os métodos de avaliação; a Seção 3 apresentará alguns estudos realizados utilizando as técnicas de autorrelato no contexto da educação; na seção 4 , será descrita a técnica Affect Grid utilizada na condução desta pesquisa; a Seção 5 apresentará as metodologias ativas utilizadas nas aulas de IHC; na Seção 6, serão descritos os passos utilizados para a execução do experimento; a Seção 7, apresentará os resultados obtidos; e na Seção 8, serão relatadas as conclusões da pesquisa.

\section{CONTEXTUALIZAÇÃO DAS TÉCNICAS DE AVALIAÇÃO DE UX}

A evolução tecnológica, o surgimento de inúmeros produtos inovadores e o crescimento da exigência dos consumidores têm levado pesquisadores e profissionais da área de UX a desenvolver métodos e técnicas que possibilitem capturar e identificar os sentimentos dos usuários nos mais diversos contextos [22].

Os Sistemas Sociais (SS) como o Twitter e o Facebook têm motivado o desenvolvimento de metodologias e técnicas para avaliar as emoções verbalizadas pelos usuários através das palavras escritas. Os métodos utilizados neste contexto são diversificados, englobando análise léxica e aprendizagem de máquina [14], rótulos de sentimentos [19], árvore de taxonomia [20] e avaliação de Postagens Relacionadas ao Uso (PRUs) [11]. O principal problema em avaliar a UX nesta área é automatizar os processos de análise das palavras.

Outra forma de avaliar a UX é por reações expressivas, tais como, expressões faciais, vocais e posturais. Estas reações geralmente acompanham a experiência emocional e permitem induzir, por exemplo, quando uma pessoa está triste, alegre, deprimida ou excitada. O principal desafio destas técnicas é melhorar os algoritmos de reconhecimento de expressões e permitir detectar as emoções de forma mais eficiente em condições ambientais adversas [9]. Os 
algoritmos de reconhecimento de expressões têm que ser imunes a variações de luz no ambiente, à presença de outras pessoas, aos movimentos bruscos, dentre outras variáveis perturbadoras.

Algumas técnicas de UX capturam dados a partir de sensores. Existe atualmente sensores para monitoramento cardíaco, monitoramento de pressão arterial, sensor de temperatura, dentre outros [10]. Os sensores, entretanto, são na maioria das vezes equipamentos invasivos, por precisarem estar conectados ao corpo humano. Esta característica pode ser um impedimento para que as técnicas baseadas em sensores sejam utilizadas em múltiplos contextos. Além disso, um avaliador de UX pode necessitar capturar diferentes tipos de emoções em uma mesma avaliação, logo seria necessário a utilização de diferentes sensores para capturar todas as emoções.

As técnicas de autorrelato são métodos que possuem um formalismo para que os próprios usuários informem seus sentimentos em relação a um produto, serviço ou sistema. Desta forma, as técnicas de autorrelato prescindem de algoritmos sofisticados para processamento automático de textos, imagens, gestos do usuário e do uso de sensores invasivos para a medição da UX. Como desvantagem, as técnicas de autorrelato são menos oportunistas, por necessitarem da colaboração do usuário para a captura das emoções.

Na próxima seção, serão descritos alguns trabalhos que utilizaram as técnicas de autorrelato para obter informações de UX no contexto educacional.

\section{AS TÉCNICAS DE AUTORRELATO APLICADAS AO CONTEXTO EDUCACIONAL}

Na literatura, existem inúmeros trabalhos $[3,5,7,16]$ que utilizam as técnicas de UX para avaliar a qualidade hedônica através de questionários, evidenciando que esta metodologia está madura e sistematizada. Estas técnicas ajudam a compreender o estado emocional do usuário sobre cada detalhe de um produto ou serviço, assim, os responsáveis em prover o produto ou serviço podem realizar ações para melhorar a UX.

No contexto educacional, alguns trabalhos foram propostos utilizando técnicas de autorrelato como ferramenta para a interlocução entre os pesquisadores e o público alvo dos estudos. Apesar de não serem encontrados muitos trabalhos neste lócus de pesquisa, podem-se destacar os seguintes:

- No estudo de [6], foi avaliada a satisfação dos usuários com os aplicativos instalados em seus smartphones levando em consideração informações contextuais e sociais. Dentre os resultados relatados na pesquisa, ressaltam-se a frequência e os tipos de aplicativos utilizados no contexto acadêmico.

- Em [23] foi estudado como os discentes, docentes e líderes escolares se envolvem com a educação e como esta interação influencia nas suas vidas pessoais.

- No trabalho de [21], foi realizado um levantamento das experiências pedagógicas mediadas por tecnologias em sala de aula e a satisfação que estas trouxeram para os discentes e docentes.

- Na pesquisa de [22], foram investigados 133 estudantes de graduação para medir o envolvimento afetivo, cognitivo e comportamental através de perguntas antes, durante e após a realização das aulas com o objetivo de encontrar padrões e diferenças de comportamento entre os estudantes, além de avaliar a sua relação com as disciplinas e professores.

Dos trabalhos listados, o que mais se assemelha com o proposto neste artigo é o [21]. Entretanto, os autores estudaram a influência das técnologias em sala de aula, enquanto a proposta deste estudo é direcionado às práticas pedagógicas independente de serem mediadas por tecnologia.

Existem dezenas de técnicas de autorrelato e suas variações. Está fora do escopo deste artigo, entretanto, uma discussão detalhada destas inúmeras técnicas. Desta forma, apresentamos na próxima seção apenas as características mais relevantes da técnicas Affect Grid, utilizada no experimento deste estudo.

\section{A TÉCNICA AFFECT GRID}

Desenvolvido por [18], o Affect Grid é uma técnica projetada como um meio rápido de avaliar o efeito ao longo das dimensões do prazer e da excitação em uma única resposta. Segundo os desenvolvedores esta técnica é potencialmente adequada para qualquer estudo que exija julgamentos sobre o afeto de um tipo descritivo ou subjetivo.

A técnica avalia duas dimensões representadas em uma matriz de $9 \times 9$ (Figura 1), onde as colunas indicam o prazer, tendo nos extremos desagradável e agradável. As linhas, representam a segunda dimensão, indicando a excitação, tendo como extremos a alta excitação e sonolência.

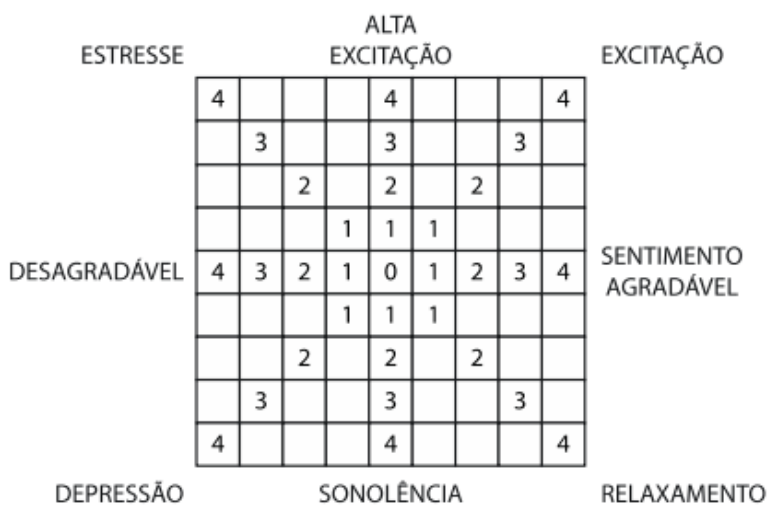

Figura 1: Técnica Affect Grid.

Como as emoções são subjetivas, esta técnica concebe um mapa de sentimentos em diferentes níveis de uma experiência hedônica, retratando que quanto mais o resultado estiver à direita, mais positivas são as emoções, e quanto mais estiver para à esquerda, mais negativas.

Segundo [17], o Affect Grid é um instrumento adequado para realizar avaliações com um grande número de participantes e a técnica se mostra mais rápida do que a utilização de questionários tradicionais. Os autores ressaltam que a técnica possibilita avaliar fluxos contínuos de respostas afetivas para uma grande diversidade de produtos e serviços.

Para calcular o peso de cada sentimento após uma célula ser marcada na matriz, as seguintes regras são utilizadas: (i) a célula central da matriz determina um estado neutro de sentimento; (ii) do ponto central para cada extremidade possui uma grandeza que varia 

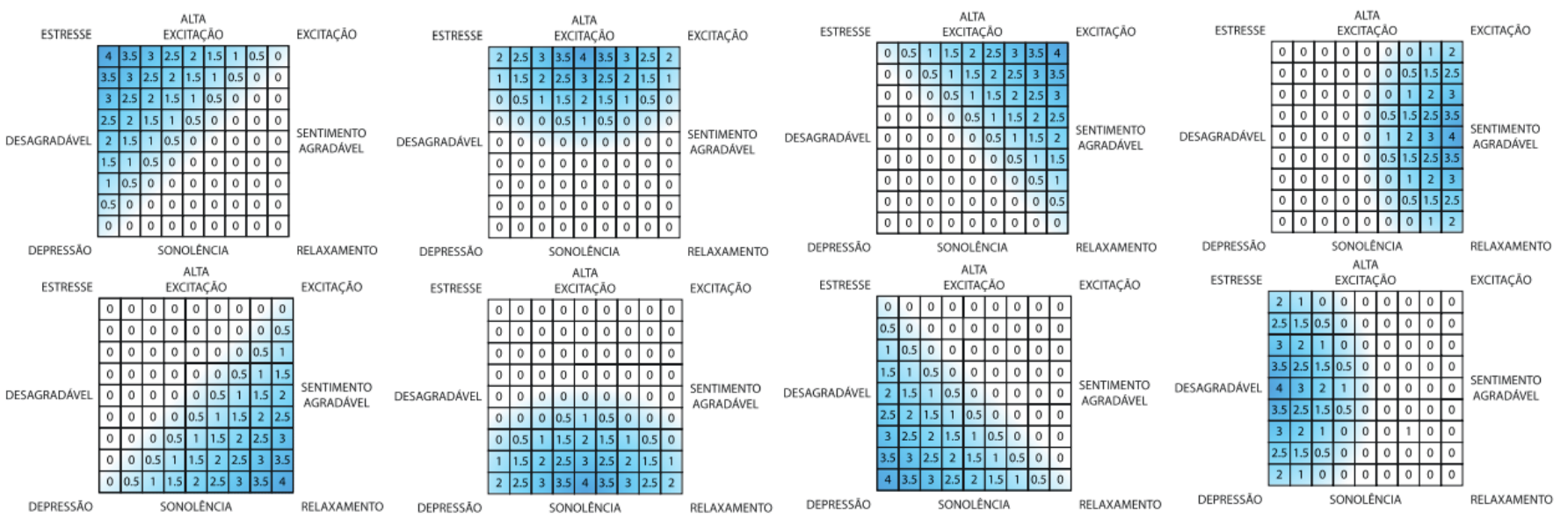

Figura 2: Distribuição dos pesos para cada sentimento na Técnica Affect Grid.

de 0 a 4, ou seja, não são admitidos valores negativos (Figura 1); (iii) para qualquer célula selecionada haverá um valor correspondente em cada sentimento (Figura 2).

Na Figura 2, são apresentados os valores para calcular o peso correspondente a cada um dos sentimentos capturados com a técnica Affect Grid. A fim de exemplificação, vamos considerar que a primeira celula da matriz seja marcada (linha $=0$, coluna $=0$ ). Neste caso, os valores seriam: Estresse $=4$, Alta Excitação $=2$, Excitação $=0$, Sentimeto Agrável $=0$, Relaxamento $=0$, Sonolência $=0$, Depressão $=0$ e Desagradável $=2$.

\section{PRÁTICAS PEDAGÓGICAS UTILIZADAS NAS AULAS DE IHC}

Nesta seção, serão apresentadas as metodologias ativas adotadas pelo professor na disciplina de IHC, em duas turmas, do curso de Engenharia da Computação, com a participação de 27 alunos.

O professor propôs diferentes práticas pedagógicas com o objetivo de avaliar as experiências hedônicas despertadas nos discentes. Segundo a definição de [1], as seguintes metodologias ativas de ensino-aprendizagem foram adotadas:

Aprendizagem por Gamificação - Este conceito aplica a mecânica de competição para engajar e motivar os estudantes a participarem das aulas. Esta metodologia foi empregada no final de conteúdos específicos abordados nas aulas e possibilitou que os alunos respondessem questionários através de uma aplicação para smartphones, específica para gamificação, denominada de Kahoot. Os três alunos com maior pontuação em cada execução desta metodologia foram bonificados com pontos extras na disciplina.

Sala Invertida - Propõe a inversão completa do modelo de ensino, capaz de engajar os alunos no conteúdo ao ponto dos mesmos lecionarem. Este método foi empregado para a abordagem de assuntos específicos da disciplina de IHC, onde todos os alunos deveriam ler previamente o conteúdo e alguns foram responsáveis em apresentálos e promover a discussão em sala de aula.

Aprendizagem Baseada em Projetos - Esta abordagem é utilizada para enfatizar as atividades e habilidades dos participantes desenvolver artefatos e aprimorar a interdisciplinaridade. As turmas avaliadas desenvolveram mapas mentais na fase de projeto de interfaces de interação e prototipação de baixa fidelidade para esboçar a resolução dos problemas propostos na disciplina.

Aprendizagem Baseada em Equipes - Corresponde a uma estratégia de ensino colaborativa aplicada em grupos. A ideia é que cada aluno tente entender individualmente os conceitos abordados em sala de aula e reforce o aprendizado pela realização de atividades colaborativas com os demais colegas. Esta metodologia foi aplicada na elaboração de protótipos de baixa fidelidade e contempla produtos semiacabados para a resolução dos problemas propostos na disciplina.

Aprendizagem Baseada em Problemas - Esta proposta defende a ideia de que a aprendizagem significativa deve ser baseada em apresentar problemas práticos para que os estudantes possam encontrar as soluções. Neste sentido, foram descritos cenários para os quais os discentes tinham que trazer soluções criativas de IHC que pudessem contornar problemas de acessibilidade, multimodalidades de interações e projetos de designer eficientes e eficazes.

Atividade Extraclasse - Este mecanismo funciona como um complemento ao conteúdo ministrado em sala de aula e permite aos estudantes praticarem e assimilarem os temas abordados. A atividade foi desenvolvida em um Ambiente Virtual de Aprendizagem (AVA) utilizando a plataforma Blackboard. Foram disponibilizadas no AVA listas de discussões, vídeos, exercícios e chats online.

\section{METODOLOGIA UTILIZADA PARA A CONDUÇÃO DO EXPERIMENTO}

Para realizar o experimento foram utilizados os procedimentos metodológicos indicados no framework DECIDE [15], cujas as ações são: (i) Determinar o objetivo da análise; (ii) Explorar perguntas a serem respondidas; (iii) Escolher o método de avaliação; (iv) Identificar e administrar as questões práticas; (v) Decidir como lidar com as questões éticas; (vi) Estabelecer forma de avaliar, interpretar e apresentar os resultados.

Com relação ao objetivo da análise, o foco do experimento é avaliar os sentimentos hedônicos despertados nos discentes com 


\begin{tabular}{|l|l|l|}
\hline ID & Perguntas & Objetivos \\
\hline \hline 1 & $\begin{array}{l}\text { Qual o seu sentimento em } \\
\text { utilizar jogos relacionados ao } \\
\text { conteúdo abordado em aula? }\end{array}$ & $\begin{array}{l}\text { Aprendizagem por Gami- } \\
\text { ficação }\end{array}$ \\
\hline 2 & $\begin{array}{l}\text { Qual o sentimento dispertado } \\
\text { ao ministrar e promover a dis- } \\
\text { cussão de conteúdos em sala } \\
\text { de aula? }\end{array}$ & Sala Invertida \\
\hline 3 & $\begin{array}{l}\text { Qual a sua avaliação quanto } \\
\text { a criação de mapas mentais e } \\
\text { protótipos? }\end{array}$ & $\begin{array}{l}\text { Aprendizagem Baseada } \\
\text { em Projetos e Aprendiza- } \\
\text { gem Baseada em Equipes }\end{array}$ \\
\hline 4 & $\begin{array}{l}\text { Como você avalia a utilização } \\
\text { de soluções criativas de IHC } \\
\text { para a solução de problemas? }\end{array}$ & $\begin{array}{l}\text { Aprendizagem Baseada } \\
\text { em Problemas }\end{array}$ \\
\hline 5 & $\begin{array}{l}\text { Qual o seu sentimento em re- } \\
\text { lação as atividades extraclasse } \\
\text { realizadas no Ambiente Vir- } \\
\text { tual de Aprendizagem (AVA)? }\end{array}$ & Atividade Extraclasse \\
\hline 6 & $\begin{array}{l}\text { Qual o seu sentimento com } \\
\text { as práticas pedagógicas utili- } \\
\text { zadas pelo professor? }\end{array}$ & Metodologias Ativas \\
\hline
\end{tabular}

Tabela 1: Perguntas elaboradas para o experimento a utilização das metodologias ativas propostas pelo professor da disciplina de IHC.

Foram especificadas seis perguntas com o propósito de avaliar os sentimentos hedônicos dos discentes em relação às metodologias utilizadas nas aulas (Tabela 1).

$\mathrm{Na}$ identificação e administração das questões práticas, foram executados os seguintes passos: (i) selecionar as turmas que participariam do experimento; (ii) apresentar a técnica de captura de UX abordada nesta pesquisa; (iii) explicar e apresentar aos estudantes informações sobre o experimento; e (iv) sistematizar a entrega, em dez aulas concecutivas, dos formulários em papel com as perguntas e uma opção de resposta no modelo da técnica Affect Grid para cada pergunta.

Em virtude do experimento necessitar do envolvimento de seres humanos, a decisão de como lidar com as questões éticas nos obriga a submeter o projeto de pesquisa ao Comitê de Ética da Universidade. Esta consulta tem a finalidade de obter a autorização para realizar a experimentação. Assim, o projeto de pesquisa foi submetido e aprovado pelo comitê de ética, e sua aprovação pode ser identificada no Parecer Consubstanciado de número (blind review).

Para avaliar e interpretar os dados, no decorrer do experimento, foram entregues aos alunos participantes as perguntas relacionadas com as metodologias aplicadas em cada aula ou em atividades extra classe. Desta forma, nem sempre as seis perguntas foram respondidas simultaneamente. Os dados foram tabulados no Microsoft Excel e os resultados serão apresentados e discutidos na próxima seção.

\section{RESULTADOS E DISCUSSÕES}

As respostas foram qualificadas em escalas variadas de intensidade, pois, na técnica Affect Grid, cada célula selecionada na grade representa um valor para cada uma das outras emoções e varia de 0 a 4 em escalas decimais conforme foi apresentado na Seção 3.

Após identificar a distância do ponto selecionado até as demais emoções, foi aplicada a média aritimética sobre os valores capturados de cada emoção e gerado um valor médio de respostas. Todas as pergunta seguiram o mesmo procedimento.

Vale ressaltar que o número de respostas para cada pergunta foi variada em função do número de alunos em sala de aula no decorrer do experimento e do número de vezes que uma determinada pergunta foi submetida, pois algumas práticas pedagógicas foram mais frequentes que outras. Desta forma, foi necessário aplicar uma segunda média aritmética com o objetivo de identificar os sentimentos hedônicos relacionados a cada metodologia aplicada em sala de aula.

Na Figura 3, são apresentados os resultados referentes à Metodologia de Gamificação e contempla os nove sentimentos capturados pela técnica Affect Grid. A analise dos resultados permite perceber que os sentimentos de Relaxamento, Sentimento Agradável e Exitação obtiveram uma pontuação muito acima dos sentimentos hedônicos negativos, como Desagrado, Depressão, Sonolência e Estresse. Desta forma, os dados indicam que as experiências hedônicas com a utilização de jogos em sala de aula geraram emoções positivas na maioria dos alunos, comprovando ser uma boa prática pedagógica para este grupo de estudantes no contexto em que foi proposto o experimento.

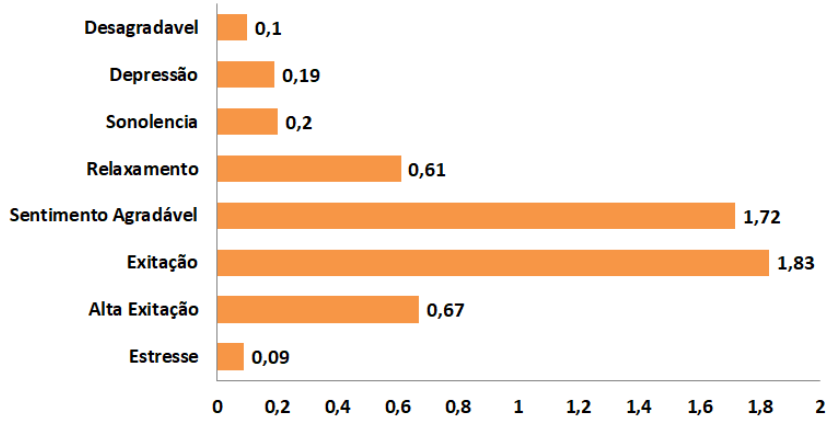

Figura 3: Resultado da avaliação da Gamificação.

Os resultados da avaliação da Sala Invertida, apresentados no gráfico da Figura 4, demonstram que a maioria dos sentimentos hedônicos positivos tiveram uma pontuação alta (Sentimento Agradável, Exitação e Relaxamento). Entretanto, ocorreu uma incidência acentuada de marcações indicando sentimentos negativos (Sonolência, Depressão e Desagradável). Analisando as possíveis causas, pode-se levar em consideração o fato dos alunos estarem em exposição acima do normal pois precisam ministrar a aula e promover a discussão do assunto como se fossem o professor, o que pode levar a um certo desconforto. Outro aspecto que pode ser levantado é o fato dos alunos não possuirem a mesma experiência do professor para manter a turma motivada e participativa, o que, em alguns casos, pode causar sonolência aos colegas. 


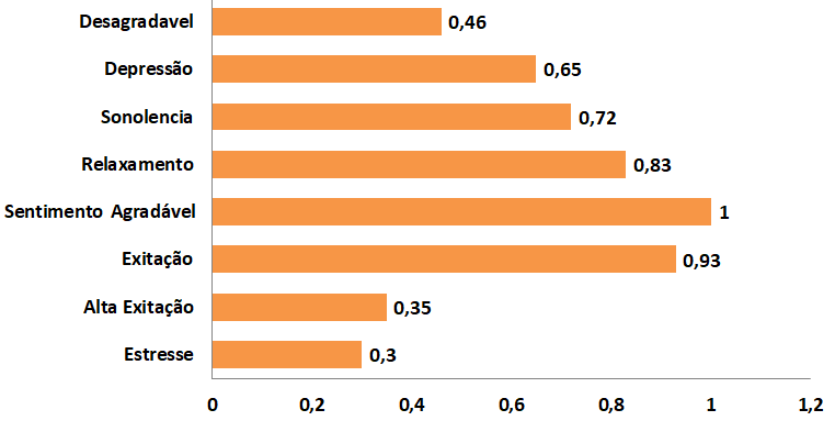

Figura 4: Resultado da avaliação da Salas Invertidas.

Os resultados obtidos com a avaliação das metodologias Baseado em Equipe e Baseado em Projeto (Figura 5) possibilitam constatar que os sentimentos Agradável e Excitação predominam na realização destas práticas pedagógicas. Além disso, é possível observar que os sentimentos hedônicos negativos são praticamente insignificantes diante dos positivos. Portanto, pode-se concluir que, de forma geral, estas metodologias atingiram os objetivos esperados pelo professor.

Outra constatação relevante é que os sentimentos de Exitação e Alta Exitação combinados apresentam uma pontuação alta. Este fato pode ser desencadeado pela motivação dos alunos trabalharem em equipe e produzirem soluções concretas para a área de IHC com utilização de modelos mentais e protótipos de baixa fidelidade.

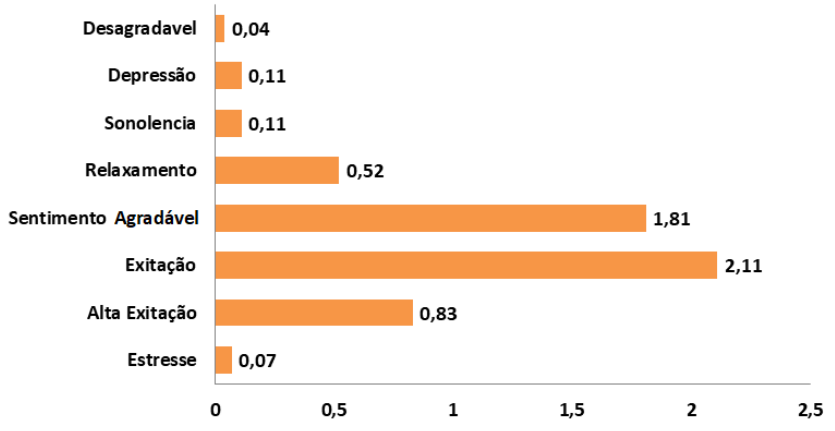

Figura 5: Resultado da metodologia Baseada em Projeto e em Equipe.

A metodologia Baseada em Problemas tinha o desafio de propor aos alunos a resolução de questões práticas de IHC como acessibilidade, multimodalidades de interações e projetos de designer eficientes e eficazes. Para resolver os problemas propostos, os alunos precisavam criar protótipos de alta fidelidade. Conforme os dados da Figura 6, é possível observar que os sentimentos hedônicos positivos (Sentimento Agradável, Excitação e Relaxamento) obtiveram as maiores pontuações. Apesar disso, os sentimentos como Sonolência e Depressão foram pontuados de forma significativa, o que pode indicar insatisfação por parte de alguns alunos por não estarem preparados para os desafios propostos ou não terem se engajado na proposta como era esperado pelo professor.

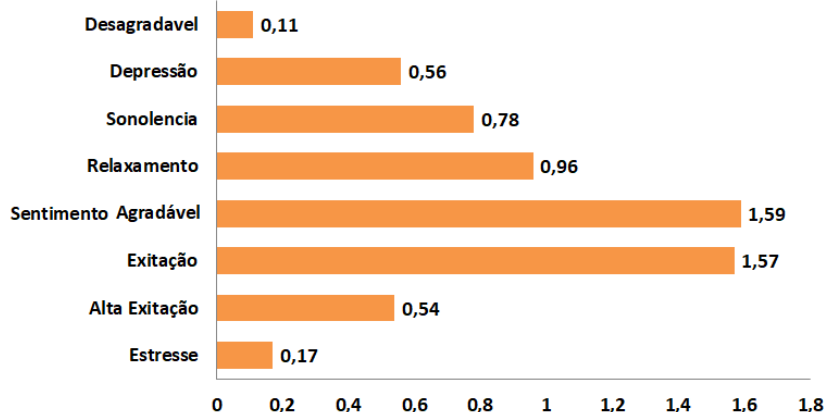

Figura 6: Resultado da Aprendizagem Baseada em Problema.

O resultado da avaliação da Atividade Extraclasse (Figura 7) foi a que demonstrou a maior pontuação para os sentimentos hedônicos negativos. Esta atividade se propós a trabalhar com o AVA, sendo disponibilizadas listas de exercícios, chats online, fóruns e vídeos. $\mathrm{O}$ objetivo do professor foi fazer com que o AVA fosse uma extensão da sala de aula e permitir o acesso de qualquer local e horário. Entretanto, para grande parte dos alunos, as atividades propostas geraram Desagrado, Depressão e Estresse. Desta forma, esta prática pedagógica deveria ser repensada.

Em um estudo realizado por [13], foi constatado que a utilização do AVA com incentivos e recompensas extrínsecas podem reduzir a ansiedade e aumentar o prazer percebido uma vez que as recompensas são consideradas importantes impulsionadores de motivações intrínsecas. Desta forma, como sugerem os autores, a inclusão de gamificação nas atividades propostas no AVA poderiam ser uma boa solução para diminuir a incidência de emoções hedônicas negativas.

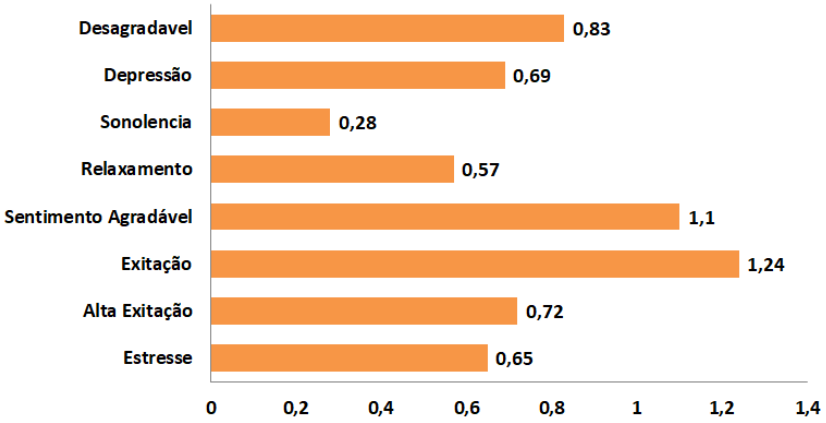

Figura 7: Resultado da Atividade Extraclasse.

Como forma de avaliar o desenho metodológico proposto pelo professor, foi sugerido aos alunos que avaliassem as práticas pedagógicas utilizadas no decorrer da disciplina. Como pode ser observado na Figura 8, os resultados indicam que os sentimentos hedônicos positivos (Agradável, Exitação, Relaxamento) se sobressaem em relação aos negativos. Estes resultados indicam que o professor escolheu um bom desenho metodológico para engajar os alunos nas suas propostas pedagógicas. 


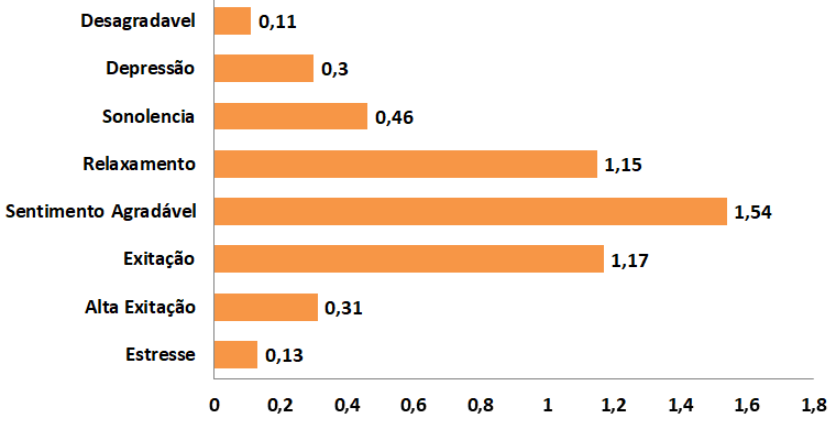

Figura 8: Resultado do desenho metodológico adotado pelo professor.

\section{CONCLUSÕES}

Diversas pesquisas estão sendo realizadas com o objetivo de analisar as potencialidades das metodologias ativas para o aprendizado ou facilidade de retenção do conhecimento por parte dos discentes. Entretanto, até o momento, existem poucos trabalhos que se proponham a avaliar a experiências dos alunos frente às metodologias ativas de ensino.

Neste artigo, foi apresentado um método para mensurar os sentimentos hedônicos envolvidos na aplicação de metodologias ativas como práticas pedagógicas, o que permite aos docentes identificar as metodologias que realmente despertam sentimentos positivos, favorecendo o engajamento dos discentes. A avaliação das metodologias ativas sob esta percepção proporciona ao professor criar os seus desenhos metodológicos com foco na satisfação do seu público alvo.

Nos resultados do experimento piloto, os alunos apresentaram sentimentos positivos para a maioria das metodologias ativas de ensino. Isto não significa que a simples adoção de metodologias ativas em sala de aula prescinde de um bom sincronismo de conteúdo e do apropriado desenho metodológico da disciplina. O entendimento da dinâmica em sala de aula e consequente adesão dos alunos estão profundamente relacionados ao sucesso da condução da disciplina. Além disso, os resultados do teste piloto não podem ser generalizados porque o público alvo e o contexto foram escolhidos por conveniência. Esta particularidade, entretanto, não inviabiliza a contribuição deste trabalho que se propõe a descrever os passos para a replicação do experimento em diferentes contextos acadêmicos e permite aos docentes de diferentes áreas avaliar as suas práticas pedagógicas sob a ótica da UX.

Como trabalhos futuros, pretende-se continuar as investigações quanto a adoção de metodologias ativas e os sentimentos hedônicos gerados aos discentes em disciplinas da área de computação que apresentam grande impacto na evasão dos cursos como, as disciplinas de cálculo e programação.

\section{REFERÊNCIAS}

[1] Aline Diesel, Alda Leila Santos Baldez, and Silvana Neumann Martins. 2017. Os princípios das metodologias ativas de ensino: uma abordagem teórica. Revista Thema 14, 1 (2017), 268-288.

[2] Angel Fidalgo-Blanco, Margarita Martinez-Nuñez, Oriol Borrás-Gene, and Javier J Sanchez-Medina. 2017. Micro flip teaching-An innovative model to promote the active involvement of students. Computers in Human Behavior 72 (2017),
713-723.

[3] Sarah E. Garcia and Laura M. Hammond. 2016. Capturing \& Measuring Emotions in UX. In Proceedings of the 2016 CHI Conference Extended Abstracts on Human Factors in Computing Systems (CHI EA '16). ACM, New York, NY, USA, 777-785. https://doi.org/10.1145/2851581.2851605

[4] Kasper Hornbæk and Morten Hertzum. 2017. Technology acceptance and user experience: A review of the experiential component in HCI. ACM Transactions on Computer-Human Interaction (TOCHI) 24, 5 (2017), 33.

[5] Eija Kaasinen, Virpi Roto, Jaakko Hakulinen, Tomi Heimonen, Jussi P. P. Jokinen, Hannu Karvonen, Tuuli Keskinen, Hanna Koskinen, Yichen Lu, Pertti Saariluoma, Helena Tokkonen, and Markku Turunen. 2015. Defining user experience goals to guide the design of industrial systems. Behaviour \& Information Technology 34, 10 (2015), 976-991. https://doi.org/10.1080/0144929X.2015.1035335 arXiv:https://doi.org/10.1080/0144929X.2015.1035335

[6] Artur H Kronbauer, Diferson Machado, and Celso AS Santos. 2015. Capture and analysis of interaction data for the evaluation of user experience with mobile devices. In International Conference of Design, User Experience, and Usability. Springer, 54-65.

[7] Hsiao-Chun Lai and Rushani Wirasinghe. 2017. Applied Research for Advertising Products: Tactics for Effective Research. In Proceedings of the 2017 CHI Conference Extended Abstracts on Human Factors in Computing Systems (CHI EA '17). ACM, New York, NY, USA, 1144-1151. https://doi.org/10.1145/3027063.3053338

[8] Effie Lai-Chong Law, Paul Van Schaik, and Virpi Roto. 2014. Attitudes towards user experience (UX) measurement. International fournal of Human-Computer Studies 72, 6 (2014), 526-541.

[9] Xiao Liu and Kiju Lee. 2018. Optimized Facial Emotion Recognition Technique for Assessing User Experience. In 2018 IEEE Games, Entertainment, Media Conference (GEM). IEEE, 1-9.

[10] Camila Loiola Brito Maia and Elizabeth Sucupira Furtado. 2016. A systematic review about user experience evaluation. In International Conference of Design, User Experience, and Usability. Springer, 445-455.

[11] Marília S Mendes, Elizabeth Furtado, Vasco Furtado, and Miguel F de Castro. 2014. How do users express their emotions regarding the social system in use? A classification of their postings by using the emotional analysis of Norman. In International Conference on Social Computing and Social Media. Springer, 229-241.

[12] Alexander G Mirnig, Alexander Meschtscherjakov, Daniela Wurhofer, Thomas Meneweger, and Manfred Tscheligi. 2015. A formal analysis of the ISO 9241-210 definition of user experience. In Proceedings of the 33rd Annual ACM Conference Extended Abstracts on Human Factors in Computing Systems. ACM, 437-450.

[13] Natália Oliveira and Sean Siqueira. 2018. Entendendo a Motivação para o Uso de um Ambiente Virtual de Aprendizagem: Um Survey com base no Modelo TAM3 e na Teoria da Autodeterminação. In Brazilian Symposium on Computers in Education (Simpósio Brasileiro de Informática na Educação-SBIE), Vol. 29. 378.

[14] Alvaro Ortigosa, José M Martín, and Rosa M Carro. 2014. Sentiment analysis in Facebook and its application to e-learning. Computers in human behavior 31 (2014), 527-541.

[15] Jenny Preece, Yvonne Rogers, and Helen Sharp. 2005. Design de interação. bookman.

[16] Claire Rowland, Elizabeth Goodman, Martin Charlier, Ann Light, and Alfred Lui. 2015. User Experience Design for the Internet of Things. , 44 pages.

[17] James A. Russell. 1980. A circumplex model of affect. Fournal of Personality and Social Psychology 39, 6 (1980), 1161-1178. https://doi.org/10.1037/h0077714

[18] James A. Russell, Anna Weiss, and Gerald A. Mendelsohn. 1989. Affect Grid: A single-item scale of pleasure and arousal. Journal of Personality and Social Psychology 57, 3 (1989), 493-502. https://doi.org/10.1037/0022-3514.57.3.493

[19] Hassan Saif, Miriam Fernandez, Yulan He, and Harith Alani. 2013. Evaluation datasets for Twitter sentiment analysis: a survey and a new dataset, the STS-Gold. (2013).

[20] Kim Schouten and Flavius Frasincar. 2016. Survey on aspect-level sentiment analysis. IEEE Transactions on Knowledge and Data Engineering 28, 3 (2016), 813-830.

[21] Virginia Thomas and Margarita Azmitia. 2016. Tapping into the app: updating the experience sampling method for the 21st century. Emerging Adulthood 4, 1 (2016), 60-67.

[22] Kui Xie, Benjamin C Heddy, and Barbara A Greene. 2019. Affordances of using mobile technology to support experience-sampling method in examining college students' engagement. Computers \& Education 128 (2019), 183-198.

[23] Sabrina Zirkel, Julie A Garcia, and Mary C Murphy. 2015. Experience-sampling research methods and their potential for education research. Educational Researcher 44, 1 (2015), 7-16. 